\title{
¿Proteínas de origen vegetal o de origen animal?: Una mirada a su impacto sobre la salud y el medio ambiente
}

\author{
Proteins of vegetable or animal origin? A look at their impact on \\ health and environment
}

\author{
Dayana Quesada 1*, Georgina Gómez' \\ Recibido: 29 de enero de 2019. Aceptado para publicación: 21 de marzo de 2019 \\ Publicado en línea, marzo 23 de 2019 \\ https://doi.org/10.35454/rncm.v2n1.063
}

\begin{abstract}
Resumen
La ingesta adecuada de proteínas resulta crucial para el óptimo funcionamiento del organismo. Según su fuente, las proteínas pueden ser de origen animal o vegetal. Las primeras son una excelente fuente de zinc, hierro hemínico, vitaminas del complejo B y aminoácidos esenciales, se digieren con más facilidad y aportan todos los aminoácidos esenciales. Sin embargo, un alto consumo de proteínas de origen animal se asocia a un mayor riesgo de mortalidad y desarrollo de complicaciones para la salud cardiovascular. Aunque las proteínas de origen vegetal suelen percibirse como una fuente de proteína incompleta al contener una menor o nula cantidad de alguno de los aminoácidos limitante, es posible obtener proteínas de alta calidad mediante la combinación de fuentes vegetales. Tanto la proteína de origen animal como vegetal son ricas en péptidos funcionales que pueden actuar como factores inmonumoduladores, antitrombóticos, e hipocolesterolémicos, entre otros. La producción de proteínas vegetales implica un impacto ambiental considerablemente menor, en comparación con la producción de proteínas de origen animal. Por consiguiente, es necesario avanzar en la producción y la promoción de alternativas de alimentos, fuente de proteína vegetal, que suplan las necesidades nutricionales de la población y disminuyan el coste para el medio ambiente asociado a la producción y consumo de proteínas de origen animal.
\end{abstract}

Palabras clave: proteínas vegetales, proteínas animales, sostenibilidad, medio ambiente.

\begin{abstract}
Summary
Adequate protein intake is crucial for the optimal functioning of the body. Depending on their source, proteins may be of animal or plant origin. The former are an excellent source of zinc, heme iron, B vitamins and essential amino acids, are more easily digested and provide all essential amino acids. However, a high intake of animal proteins is associated with an increased risk of mortality and development of cardiovascular health complications. Although plant-based proteins are often perceived as an incomplete protein source because they contain little or no limiting aminoacids, it is possible to obtain high quality proteins from a combination of plant sources. Both animal and vegetable proteins are rich in functional peptides that can act as an immune modulating, antithrombotic, hypocholesterolemic and other factors. The production of vegetable proteins implies a considerably smaller environmental impact, in comparison with the production of proteins of animal origin. Therefore, it is necessary to advance in the production and promotion of alternative foods, a source of vegetable protein, that supply the nutritional needs of the population and reduce the cost to the environment associated with the production and consumption of animal protein.
\end{abstract}

Keywords: Vegetable proteins; Animal proteins; Sustainable development; Environment.

1. Departamento de Bioquímica, Escuela de Medicina, Universidad de Costa Rica. San José, Costa Rica.

*Correspondencia: Dayana Quesada

dahiana.quesada37@gmail.com 


\section{INTRODUCCIÓN}

Es bien sabido que los patrones de alimentación tienen un impacto significativo tanto en la salud como en el medio ambiente. Recientemente se ha originado una amplia discusión con respecto a la sostenibilidad de la producción de los alimentos que se consumen, dado que la producción, el mercadeo y el consumo de las diferentes fuentes de nutrientes de la dieta tienen diferentes efectos sobre la biodiversidad, el uso del agua y de la tierra, el cambio climático, la salud humana y el bienestar animal ${ }^{(1)}$. De acuerdo con la Organización de Naciones Unidas para la Alimentación y la Agricultura (FAO) una dieta sostenible es aquella nutricionalmente adecuada, saludable, segura, con bajo impacto sobre el medio ambiente, económicamente accesible y culturalmente aceptable ${ }^{(2)}$. Sin embargo, la selección de alimentos está determinada por diversos factores culturales y socioeconómicos que pueden alejar las prácticas alimentarias de esta definición ${ }^{(3)}$. En el caso de las proteínas, por ejemplo, se ha hecho énfasis acerca del impacto negativo de la producción y el consumo de la proteína animal, tanto para la salud humana como para el medio ambiente; sin embargo, su consumo ha aumentado, de manera evidente, en las últimas décadas ${ }^{(4)}$. Este artículo se propone comparar los beneficios del consumo de proteínas para la salud humana y el impacto ambiental, según su fuente: proteínas de origen animal o vegetal.

\section{CARACTERÍSTICAS GENERALES DE LAS PROTEÍNAS}

Las proteínas son las macromoléculas orgánicas más abundantes en las células vivas, y en el ser humano. Se desempeñan como componentes estructurales, enzimas, hormonas, mensajeros, transportadores y componentes del sistema inmune, entre otras. Están construidas a partir un mismo conjunto de 20 aminoácidos, de los cuales nueve no pueden ser sintetizados en el organismo, por lo que se les considera aminoácidos esenciales ${ }^{(5)}$.

La capacidad del organismo para sintetizar nuevas proteínas depende, entre otros aspectos, de la disponibilidad de aminoácidos esenciales. El valor biológico o la calidad de la proteína dietética está determinado fundamentalmente por la composición y la disponibilidad de los aminoácidos esenciales, que podría verse afectada por factores como el estado fisiológico y la edad del organismo $^{(6)}$. El valor biológico es máximo cuando las proporciones de aminoácidos esenciales son suficientes para satisfacer las demandas de nitrógeno y aminoácidos para el crecimiento, la síntesis y reparación tisular, tomando como referencia las necesidades proteicas de niños mayores de un año de edad. La digestibilidad de las proteínas es otro de los factores críticos en la evaluación de la calidad de estas ${ }^{(7)}$. La digestibilidad de las proteínas es definida como la relación entre el nitrógeno consumido respecto al absorbido. Se considera una digestibilidad $=100$ (igual a 100) cuando la totalidad del nitrógeno absorbido por el organismo equipara al consumido. Uno de los métodos para la estimación de este valor es el análisis del contenido de nitrógeno presente en las heces ${ }^{(6)}$; sin embargo, esta prueba asume que todos los aminoácidos tienen la misma digestibilidad o que el aminoácido es absorbido en las mismas proporciones, indistintamente de la porción o tracto del intestino delgado ${ }^{(8)}$. Las investigaciones desarrolladas por Mathai, Schaafsma et al., coinciden con lo propuesto por la Organización de las Naciones Unidas para la Agricultura y la Alimentación, en que el íleon parece ser la porción más fiable para la determinación de dicha evaluación ${ }^{(7-9)}$.

Para la evaluación de la calidad de las proteínas se cuenta con dos métodos estandarizados: Puntaje Corregido por Digestibilidad de la proteína (Protein digestibility corrected amino acid score PDCAAS) y el Puntaje Digestible de los Aminoácidos Esenciales (Digestible indispensable amino acid score DIAAS) ${ }^{(10)}$. La principal diferencia entre los métodos PDCAAS y DIIAS es que el primero asume que la digestibilidad del aminoácido es la misma en todo el intestino, mientras que el DIAAS utiliza como referencia la digestibilidad en el íleon. Además, la digestibilidad para el PDCAAS ha sido probada en ratas y la del DIAAS en cerdos, que parece ser un modelo más aceptado ${ }^{(8)}$.

Junto a lo anterior, la calidad de la proteína puede verse afectada por diversos factores. Las proteínas de origen animal, por ejemplo, se digieren con mayor facilidad que las de origen vegetal, ya que estas últimas por lo general, están almacenadas en gránulos, lo que las hacen menos accesibles a las enzimas digestivas. Algunas plantas contienen inhibidores de las enzimas digestivas, sin embargo, estos se inactivan con el calor, lo que lleva a un aumento de la digestibilidad con el proceso de cocción $^{(11)}$. La presencia de factores intrínsecos de la pared celular, factores "anti nutricionales" como los polifenoles o el contenido de fibra dietética y o reacciones químicas que se generen en el proceso de digestión también pueden alterar la digestibilidad de las proteínas ${ }^{(10)}$.

En general, los alimentos de origen animal obtienen mayores valores de PDCAAS y DIAAS, independiente 
del método de análisis, lo cual se asocia con una mejor calidad de la proteína ${ }^{(12)}$. El PDCAAS se calcula dividiendo la cantidad ( $\mathrm{mg}$ ) del aminoácido limitante en la proteína a evaluar entre la cantidad ( $\mathrm{mg}$ ) del mismo aminoácido en la proteína de referencia, que usualmente es el huevo ${ }^{(13)}$. Siguiendo lo establecido por la FAO, respecto a los patrones recomendados de aminoácidos, los resultados descritos por Mathai et al. indican que las proteínas lácteas resultan ser excelentes fuentes de proteína, con un valor DIIAS $\geq 100^{(8)}$. Aunado a lo anterior, los alimentos de origen animal (carnes de res, cerdo, aves, pescado, mariscos, huevos y lácteos) además de constituir una buena fuente de proteína y aminoácidos esenciales, son fuentes importantes de cobalamina o vitamina B12, zinc, fósforo, y de hierro hemínico, este último es absorbido en $15 \%$ - $40 \%$ en comparación con el hierro no hemínico presente en las plantas (1\%-15\%). De igual manera, el zinc contenido en las carnes es mejor absorbido que el presente en las plantas, debido a la presencia de componentes anti nutricionales en estas como oxalatos, fitatos y taninos que pueden disminuir su absorción ${ }^{(5)}$.

Respecto a las proteínas de origen vegetal, estas suelen percibirse como una fuente de proteína incompleta al contener una menor o nula cantidad de alguno de los aminoácidos esenciales, al que se le denomina aminoácido limitante ${ }^{(12)} \mathrm{y}$ con un PDCAAS significativamente más bajo, entre 0,5 y $0,75^{(14)}$. A pesar de ello, un análisis desarrollado por Suárez López, y colaboradores ${ }^{(6)}$ identificó que alimentos de origen vegetal como la remolacha (betabel), los garbanzos, el pistacho y la soya no contienen aminoácidos limitantes y obtuvieron valores de PDCAAS superiores a 0,75 (Tabla 1). De igual manera, el aislado de proteína de soya y la harina de soya se han clasificado como proteína de buena calidad (DIIAS $\geq 75 \mathrm{y}<100)^{(6)}$; más, a la proteína de arvejas y el trigo se le asignan puntajes de $62 \%$ y $45 \%$ DIIAS, respectivamente, que de acuerdo con lo propuesto por la FAO se considerarían proteínas de baja calidad ${ }^{(9)}$.

$\mathrm{El}$ análisis de la composición de cereales como el trigo, el mijo y el maíz, coinciden en que, la cisteína, metionina, treonina y triptófano son los que se presentan en mayores cantidades, mientras que la lisina es el aminoácido limitante; dichos análisis se han aplicado a alimentos como el trigo, el mijo y el maíz ${ }^{(15,16)}$. En las leguminosas se identificaron como principales aminoácidos limitantes la metionina y la cisteína ${ }^{(16)}$. Woolf $e t$ al. ${ }^{(13)}$ también reportan deficiencias en metionina en las nueces de macadamia y las frutas como el durazno y la cereza. Las nueces de Brasil, presentan un alto conte- nido de metionina mientras que el pepino "sin cáscara" y los duraznos se destacan por su aporte de triptófano.

Tabla 1. Puntaje obtenido para ESCORE, PDCAAS y aminoácidos limitantes en proteínas animales y vegetales.

\begin{tabular}{|c|c|c|c|}
\hline Alimento & ESCORE & PDCAAS & Aa* Limitante \\
\hline \multicolumn{4}{|c|}{ Fuentes de origen animal } \\
\hline Huevo & 100 & 97 & No tiene \\
\hline Leche & 100 & 95 & No tiene \\
\hline Queso & 100 & 95 & No tiene \\
\hline Atún & 100 & 94 & No tiene \\
\hline Carnes (promedio) & 100 & 94 & No tiene \\
\hline \multicolumn{4}{|c|}{ Fuentes de origen vegetal } \\
\hline Remolacha & 100 & 83,0 & No tiene \\
\hline Garbanzos & 100 & 78,0 & No tiene \\
\hline Soja & 100 & 78,0 & No tiene \\
\hline Espinaca & 90,4 & 75,0 & Azufrados \\
\hline Zanahoria & 89,6 & 74,4 & Lisina \\
\hline Arvejas & 95,2 & 74,3 & Azufrados \\
\hline Judías (chauchas) & 88,8 & 73,7 & Azufrados \\
\hline Pistacho & 100 & 73,0 & No tiene \\
\hline Manzana & 85,2 & 72,4 & Azufrados \\
\hline Coco & 83,3 & 70,8 & Lisina \\
\hline Papas & 85,0 & 70,5 & Histidina \\
\hline Espárragos & 79,5 & 65,9 & Leucina \\
\hline Lentejas & 81,2 & 63,3 & Azufrados \\
\hline Granos de Girasol & 70,6 & 60,7 & Lisina \\
\hline
\end{tabular}

Calidad de las proteínas según el método PDCAAS y aminoácido limitante en algunos alimentos de origen vegetal y animal, propuestos en el estudio de Suárez ${ }^{(15)}$.

*Aminoácido **mg/gramo de proteína

\section{COMPLEMENTARIEDAD DE LAS PROTEÍNAS}

Diversos estudios, incluyendo aquellos realizados en modelos animales, han demostrado que las proteínas tienen la capacidad de complementarse entre $\mathrm{si}^{(5,16-17)}$, por lo que en la actualidad, los complementos de proteínas vegetales son consideradas una opción saludable que permite alcanzar las recomendaciones de ingesta establecidas para este macronutriente ${ }^{(18)}$. Woolf et al. ${ }^{(13)}$ 
recomiendan considerar las propiedades de cada alimento, de manera que las proporciones requeridas para una óptima complementación puedan definirse. Estos autores identificaron una serie de combinaciones de alimentos de origen vegetal, que hacen posible obtener proteínas completas y a su vez reducir el peso del producto final, las calorías consumidas o maximizar la eficiencia de la proteína. La Tabla 2 muestra algunas de las mezclas propuestas.

Tabla 2. Combinaciones de proteínas completas a partir de alimentos de origen vegetal

\begin{tabular}{|l|l|l|}
\hline Meta & \multicolumn{1}{|c|}{ Alimento 1 } & \multicolumn{1}{c|}{ Alimento 2 } \\
\hline \multicolumn{2}{|c|}{ Minimizar el aporte energético/calórico } \\
\hline & Espirulina (Alga) & Aislado de proteína de soya \\
\hline & Harina de sésamo & Aislado de proteína de soya \\
\hline & Espirulina & Hojas de calabaza \\
\hline Maximizar la eficacia & Granos de maíz & Tomates \\
\hline & Manzana & Coco \\
\hline & Jugo de naranja & Edamame \\
\hline Disminuir el peso de la mezcla & \\
\hline & Nueces de Brasil & Aislado de proteína de soya \\
\hline & Harina de maní & Aislado de proteína de soya \\
\hline & Harina de sésamo & Aislado de proteína de soya \\
\hline
\end{tabular}

Combinaciones de alimentos de origen vegetal que aportan todos los aminoácidos esenciales, maximizan la eficacia de la proteína, disminuyen el aporte energético y el peso de la mezcla, propuestas en el estudio de Woolf ${ }^{(16)}$.

\section{PROPIEDADES BIOLÓGICAS DE LAS PROTEÍNAS DE ORIGEN VEGETAL}

Aunado a las funciones nutricionales de las proteínas, en estas macromoléculas se han identificado componentes o péptidos que resultan de la digestión gastrointestinal o por la fermentación microbiana de la proteína. Estos péptidos son capaces de ejercer funciones benéficas para el organismo, por lo que se denominan péptidos bioactivos ${ }^{(19-21)}$.

Los péptidos bioactivos son fragmentos específicos, usualmente compuestos por unidades entre 2 y 20 aminoácidos, con masas moleculares inferiores a los $6000 \mathrm{Da}$, inactivos en el interior de la proteína precursora, que ejercen su actividad biológica tras su liberación enzimática o la hidrólisis química ${ }^{(18,22-24)}$. Estas moléculas son capaces de atravesar la barrera epitelial pudiendo accionar en el nivel local, gastrointestinal o sistémico ${ }^{(24)}$. Los péptidos funcionales pueden obtenerse tanto de las proteínas de origen animal como vegetal y pueden actuar como factores opiáceos, inmonumoduladores, antitrombóticos, hipocolesterolémicos, antiinflamatorios antimicrobianos, antihipertensivos, vasorreguladores, antioxidantes, inductores hormonales, neurotransmisores, entre otros ${ }^{(22-24)}$.

En las proteínas de origen animal, los precursores de péptidos más estudiados son los presentes en la leche y sus subproductos, por ejemplo, el huevo, la carne de cerdo, algunos tejidos de pescado y los péptidos con actividad opioide $^{(24)}$.

En particular, en alimentos de origen vegetal, las secuencias Tyr-Pro-Phe-Val y Tyr-Gli-Gli-Trp-Leu presentes en la soja y el trigo, respectivamente, se han identificado o se han asociados a propiedades opiáceas. La secuencia Arg-Ile-Tyr presente en la colza tiene propiedades como supresora del apetito ${ }^{(23)}$.

García et al. ${ }^{(19)}$ describieron en forma amplia los péptidos hallados en alimentos de origen vegetal con propiedades antihipertensivas y sus secuencias respectivas. Encontraron que los mejores péptidos con esta característica contenían aminoácidos hidrofóbicos como la prolina o cargados positivamente como la lisina o arginina, de manera especial en la posición del extremo carboxilo. Algunos de los alimentos en los que estos péptidos se encuentran de manera natural son el ajo, el brócoli, los hongos comestibles y las algas ${ }^{(22)}$.

La soya contiene péptidos bioactivos con capacidad antioxidante, esto atribuido principalmente a la secuencia Leu-Leu-Pro-His-His, siendo el sitio activo el segmento Pro-His-His. Junto a esto, ya ha sido previamente descrito, que los péptidos que contienen histidina actúan como quelantes de metales, captadores de radicales hidroxilo y especies reactivas de oxígeno ${ }^{(19)}$. Gallegos y colaboradores también han descrito secuencias con capacidad antioxidante en el endospermo del arroz Fen-Arg-Asp-Glu-His-Lis-Lis y Lis-His-Asp-Arg-GliAsp-Glu-Fen, en el garbanzo Asn-Arg-Tir-His-Glu, en la colza Pro-Ala-Gli-Pro-Fen y en el trigo serrano Trp-ProLeu;Val-Pro-Trp; Val-Fen-Pro-Trp y Pro-Trp-Trp. Otras de las fuentes dietéticas de proteínas vegetales con péptidos bioactivos son las semillas de maní, girasol, hojas de alfalfa o curry, y tubérculos como el Yam ichyoimo, hongos medicinales, el amaranto y piñón mexicano; sin embargo, estas secuencias no han sido reportadas ${ }^{(19,23)}$. 


\section{CONSUMO DE PROTEÍNAS Y DESARROLLO DE ENFERMEDADES CRÓNICAS NO TRANSMISIBLES}

Si bien las proteínas de origen animal se caracterizan por su mayor digestibilidad y calidad, su consumo se ha relacionado con un aumento en el riesgo de sufrir enfermedades cardiovasculares ${ }^{(18,25-27)}$, las cuales de acuerdo con la Organización Mundial de la Salud constituyen la mayoría de las muertes por enfermedades no transmisibles, afectando más de 17,9 millones de personas al año ${ }^{(28)}$.

Los resultados de estudios prospectivos de cohorte en Estados Unidos, encontraron que, en personas con al menos un factor de riesgo (sedentarismo, fumador, exceso de peso o consumo de alcohol), el consumo de proteínas de origen animal incrementó el riesgo de mortalidad por enfermedad cardiovascular en $10,8 \%$, mientras que el mayor consumo de proteínas de origen vegetal redujo la mortalidad por todas las causas en $9 \%$; estos resultados se obtuvieron ajustando los datos por factores dietéticos y del estilo de vida ${ }^{(17,27)}$. Además, el consumo de proteínas de origen vegetal se asocia a una menor presión arterial y menores niveles de colesterol $\mathrm{LDL}^{(18,25-27)}$.

Tras 26 años de seguimiento, Bernstein et al. ${ }^{(25)}$ reportaron que el consumo de carnes rojas se asoció a un mayor riesgo de hipertensión arterial, Diabetes Mellitus tipo 2 (DMT2), hipercolesterolemia. Ajustado por edad, el consumo de proteínas vegetales significó un menor riesgo cardiovascular, el cual podría estar asociado tanto al tipo como a la cantidad de grasa característica de las fuentes respectivas de proteína. Los resultados de este estudio estimaron que el consumo de una porción diaria de nueces disminuye en un $30 \%$ el riesgo de enfermedad cardiovascular, al compararla con una porción diaria de carne roja. De manera similar, una porción de lácteos, pollo y pescado, disminuyó el riesgo de presentar esta condición en un $15 \%, 19 \%$ y $24 \%$ respectivamente ${ }^{(29)}$.

Las dietas altas en proteína han demostrado ser efectivas en la prevención del riesgo metabólico y de la DMT2 $2^{(30)}$, sin embargo, junto a la cantidad de proteína, la fuente de esta tiene un papel importante en la prevención. Un estudio en las Islas Mediterráneas demostró que el consumo de proteína animal estaba asociado a un mayor riesgo de desarrollo de DMT2, mientras que el consumo de proteínas de origen vegetal constituyó un factor protector ${ }^{(30)}$. La revisión sistemática conducida por Tian ${ }^{(30)}$ concluye que el consumo de carnes rojas o procesadas incrementa el riesgo de desarrollar DMT2, en ambos sexos, al igual que el mayor consumo de soya se relaciona con un menor riesgo de esta condición en las mujeres. El huevo y el pescado no correlacionaron en ninguna dirección con la aparición de esta condición. Los lácteos bajos en grasa y el yogurt se asociaron a un menor riesgo de desarrollo de la DMT2 ${ }^{(30)}$.

Se ha encontrado una relación dosis-respuesta para el riesgo de cáncer de mamá en mujeres que consumen proteína de soya (reducción de $9 \%$ ), leche descremada (reducción de $4 \%$ ) y carnes procesadas (incremento de $9 \%)^{(31)}$. La densidad mineral ósea también se ha visto asociada al consumo de proteína, a pesar de que los resultados no son concluyentes. Un importante estudio observacional desarrollado en el Reino Unido, encontró una asociación positiva entre el consumo de proteína y la densidad mineral ósea, tanto en hombres como en mujeres ${ }^{(32)}$; es necesario desarrollar más estudios para esclarecer el efecto que podría tener la fuente de la proteína ${ }^{(32,33)}$.

\section{IMPACTO AMBIENTAL}

Actualmente, en las dietas occidentales, la proporción consumida de proteína de origen animal versus proteína de origen vegetal está causando serios daños al ambiente, dado el alto impacto que la producción de ganado vacuno, porcino, aviar y la acuacultura, producen en la emisión de gases tipo invernadero en comparación con la producción de alimentos fuente de proteínas de origen vegetal ${ }^{(1,34)}$.

En general, la evidencia apunta a que los alimentos de origen vegetal tiene un menor efecto ambiental por unidad de peso, tamaño de la porción, gramos de proteína o energía aportada que los alimentos de origen animal, por lo que una dieta con una mayor proporción de alimentos de origen vegetal conferiría mayores beneficios no solo para la salud, sino también para el medio ambiente ${ }^{(35)}$.

A pesar de que estas carnes constituyen una valiosa fuente de nutrientes en la dieta, proporcionando proteína de alta calidad y micronutrientes esenciales, su producción es mucho menos sostenible que otras fuentes de proteína.

Se ha introducido también el término de proteínas pro-ambiente, que se refiere a la proteína producida utilizando la menor cantidad de recursos naturales de manera que se reduzca el impacto ambiental ${ }^{(36)}$. Estos conceptos han contribuido a un mejor entendimiento de la importancia de la adopción de dietas, que además de saludables sean sostenibles.

De acuerdo con de Boer y Aiking ${ }^{(34)}$, la disponibilidad de proteínas en el mundo solo puede asegurarse a través de una transición hacia un mayor consumo de proteínas 
vegetales y una disminución considerable del consumo de proteínas animales, reemplazando, por ejemplo, las carnes por leguminosas. Sin embargo, dada la gran popularidad de las carnes, dicha transición requeriría un gran esfuerzo de parte de algunos sectores de la población para poder lograr concientizar a los consumidores y productores acerca de la necesidad de este cambio ${ }^{(5)}$. Es importante tener en cuenta que, para que dicho cambio sea efectivo se deben considerar otros aspectos que influyen en la conducta alimentaria, entre ellos, los factores culturales, culinarios, económicos y geográficos propios de los diversos grupos poblacionales ${ }^{(34,37)}$.

\section{OTRAS ALTERNATIVAS PARA EL CONSUMO DE PROTEÍNAS}

Alexander et al. ${ }^{(38)}$ presentan algunas fuentes alternativas de proteína cuya producción permite una mejor utilización de los suelos y una menor producción de gases tipo invernadero, como la carne cultivada, la carne de imitación y los insectos. Los insectos comestibles, por ejemplo, tienen un gran potencial para convertir la biomasa en proteína y energía, son fuente de grasa, proteína y micronutrientes y pueden ser producidos con un bajo impacto ambiental y menor consumo de agua. Otra alternativa que está en desarrollo es la carne cultivada, esta es producida de manera sintética a partir de células madre que se diferencian en células musculares, sin embargo, su producción a gran escala se estima estará en marcha a partir del año 2021, y su éxito estará sujeto a que los consumidores acepten su sabor, apariencia y textura ${ }^{(38)}$. Más recientemente, se están desarrollando productos de imitación a base de proteínas vegetales texturizadas y proteínas derivadas de hongos ${ }^{(39)}$.

La capacidad de producir suficientes alimentos en el futuro podría verse limitada por la disponibilidad de agua, la fertilidad del suelo y el uso de las tierras y los océanos. Existe suficiente evidencia del impacto que la producción de alimentos ejerce sobre el medio ambiente al drenar los recursos naturales. Este impacto debe ser sopesado con su aporte nutricional y sus beneficios para la salud, mediante el uso responsable de los recursos naturales, con el fin de asegurar la disponibilidad de proteínas $y$ otros nutrientes para las futuras generaciones. Willet et al. ${ }^{(35)}$ sugieren que el consumo mundial de frutas, vegetales, frutos secos y leguminosas debe duplicarse, mientras que el consumo de carnes rojas y azúcar deberá reducirse a la mitad para lograr una dieta saludable y sostenible con el medio ambiente ${ }^{(35,40,41)}$.

\section{CONCLUSIONES}

Las proteínas son macromoléculas fundamentales para el óptimo funcionamiento del organismo. Como parte de su estructura, contienen péptidos bioactivos con propiedades beneficiosas para la salud. Las proteínas de origen animal se digieren más fácilmente y aportan todos los aminoácidos esenciales, sin embargo, es posible alcanzar los requerimientos nutricionales mediante la combinación de diversas proteínas de origen vegetal. Un mayor consumo de estas proteínas se asocia a un riesgo menor de mortalidad y desarrollo de complicaciones para la salud cardiovascular. En relación con la producción de alimentos, las proteínas obtenidas de fuentes animales representan una mayor huella ecológica con un impacto negativo para los recursos ambientales. Es necesario conducir a la población hacia un mayor consumo de proteínas de origen vegetal, asegurando combinaciones que permitan alcanzar las recomendaciones nutricionales.

\section{Declaración de autoría}

Las autores declaran haber trabajado en forma igualitaria en la elaboración del artículo.

\section{Conflicto de intereses}

Los autores declaran no tener conflicto de intereses.

\section{Financiamiento}

Esta revisión fue parcialmente financiada por Baltimore Spice Central America Sociedad/ Kerry, Costa Rica.

\section{Referencias bibliográficas}

1. Hartmann C, Siegrist M. Consumer perception and behaviour regarding sustainable protein consumption: A systematic review. Trends Food Sci Technol. 2017; 61:11-25.

2. Organización de las Naciones Unidas para la Alimentación y la Agricultura-FAO. Informe final. Simposio científico internacional "Biodiversidad y dietas sostenibles unidos contra el hambre”. [Internet]. Roma:3-5 de noviembre de 2010. [ Fecha de consulta:1 marzo 2019 ]. Disponible en:http://www.fao.org/ag/humannutrition/285090e85170814dd369bbb502e1128028978d.pdf

3. Vainio A, Niva M, Jallinoja P, Latvala T. From beef to beans: Eating motives and the replacement of animal proteins with plant proteins among Finnish consumers. Appetite. 2016;106:92-100. 
4. Macdiarmid JI, Douglas F, Campbell J. Eating like there's no tomorrow: Public awareness of the environmental impact of food and reluctance to eat less meat as part of a sustainable diet. Appetite. 2016;96:487-93.

5. Bohrer BM. Review: Nutrient density and nutritional value of meat products and non-meat foods high in protein. Trends Food Sci Technol. 2017;65:103-12.

6. Suárez López MM, Kizlansky A, López LB. Evaluación de la calidad de las proteínas en los alimentos calculando el escore de aminoácidos corregido por digestibilidad. Nutr Hosp. 2006;21(1):47-51.

7. Schaafsma G. Advantages and limitations of the protein digestibility-corrected amino acid score (PDCAAS) as a method for evaluating protein quality in human diets. Br J Nutr. 2012;108 Suppl 2:S333-6.

8. Mathai JK, Liu Y, Stein HH. Values for digestible indispensable amino acid scores (DIAAS) for some dairy and plant proteins may better describe protein quality than values calculated using the concept for protein digestibility-corrected amino acid scores (PDCAAS). Br J Nutr. 2017;117(4):490-9.

9. Food and Agriculture Organization of the United Nations (FAO). Dietary protein quality evaluation in human nutrition. Report of an FAO Expert Consultation. FAO Food Nutrition Paper 92. [Internet]. Roma: 2013 [Fecha de consulta enero 17 de 2019]. Disponible en: http://www.fao.org/ ag/humannutrition/35978-02317b979a686a57aa4593304ff c17f06.pdf

10. Huang S, Wang LM, Sivendiran T, Bohrer BM. Review: Amino acid concentration of high protein food products and an overview of the current methods used to determine protein quality. Crit Rev Food Sci Nutr. 2017;1-6.

11. Mahan LK, Raymond JL. Dietoterapia. 14 Edición. España: Krause; 2017.

12. de Gavelle E, Huneau J-F, Bianchi CM, Verger EO, Mariotti F. Protein Adequacy Is Primarily a Matter of Protein Quantity, Not Quality: Modeling an Increase in Plant:Animal Protein Ratio in French Adults. Nutrients. 2017;9(12): pii:E1333.

13. Grooper SS, Groff, JL, Smith JL. Advanced nutrition and human metabolism. Quinta edición. Belmont: Wadsworth Cengage Learning; 2009.

14. Marangoni F, Corsello G, Cricelli C, Ferrara N, Ghiselli A, Lucchin $\mathrm{L}$, et al. Role of poultry meat in a balanced diet aimed at maintaining health and wellbeing: an Italian consensus document. Food Nutr Res. 2015;59(1):27606.

15. Woolf PJ, Fu LL, Basu A. vProtein: identifying optimal amino acid complements from plant-based foods. PLoS One. 2011;6(4):e18836.

16. Caire-Juvera G, Vázquez-Ortiz FA, Grijalva-Haro MI. Amino acid composition, score and in vitro protein digestibility of foods commonly consumed in Norhwest Mexico. Nutr Hosp. $2013 ; 28(2): 365-71$.
17. Mariotti F. Plant Protein, Animal Protein, and Protein Quality. En: Mariotti F. Vegetarian and Plant-Based Diets in Health and Disease Prevention. Elsevier; 2017. 621-642.

18. Richter CK, Skulas-Ray AC, Champagne CM, KrisEtherton PM. Plant Protein and Animal Proteins: Do They Differentially Affect Cardiovascular Disease Risk? Adv Nutr. 2015;6(6):712-28.

19. Gallegos Tintoré S, Chel Guerrero L, Corzo Ríos LJ, Martínez Ayala AL. Péptidos con actividad antioxidante de proteínas vegetales. En: Segura Campos M, Chel Guerrero L, Betancur Ancona D. Bioactividad de péptidos derivados de proteínas alimentarias. Primera edición. OmniaScience; 2013. 111-22.

20. Zhang H, Hu C-AA, Kovacs-Nolan J, Mine Y. Bioactive dietary peptides and amino acids in inflammatory bowel disease. Amino Acids. 2015;47(10):2127-41.

21. Rodríguez-Hernández G, Rentería-Monterrubio AL, RodríguezFigueroa JC, Chávez-Martínez A. Biopéptidos en la leche y sus derivados: funcionamiento y beneficios a la salud. Ecosistemas y Recur Agropecu. 2014;1(3):281-94.

22. García MC, Puchalska P, Esteve C, Marina ML. Vegetable foods: A cheap source of proteins and peptides with antihypertensive, antioxidant, and other less occurrence bioactivities. Talanta. 2013;106:328-49.

23. Sarmadi BH, Ismail A. Antioxidative peptides from food proteins: A review. Peptides. 2010;31(10):1949-56.

24. Mulero Cánovas J, Zafrilla Rentero P, Martínez-Cachá Martínez A, Leal Hernández M, Abellán Alemán J. Péptidos bioactivos. Clínica e Investig en Arterioscler. 2011;23(5):219-27.

25. Sluijs I, Beulens JWJ, van der A DL, Spijkerman AMW, Grobbee DE, van der Schouw YT. Dietary Intake of Total, Animal, and Vegetable Protein and Risk of Type 2 Diabetes in the European Prospective Investigation into Cancer and Nutrition (EPIC)-NL Study. Diabetes Care. 2010;33(1):43-8.

26. Pino L Á, Cediel G G, Hirsch B S. Ingesta de alimentos de origen animal versus origen vegetal y riesgo cardiovascular. Rev Chil Nutr. 2009;36(3):210-6.

27. Song M, Fung TT, Hu FB, Willett WC, Longo VD, Chan AT, et al. Association of Animal and Plant Protein Intake With All-Cause and Cause-Specific Mortality. JAMA Intern Med. 2016;176(10):1453-63.

28. Organización Mundial de la Salud. Enfermedades no transmisibles [Internet]. OMS. 1 de junio de 2018. [Fecha de consulta: 1 de marzo 2019 ]. Disponible en: https://www.who. int/es/news-room/fact-sheets/detail/noncommunicablediseases

29. Bernstein AM, Sun Q, Hu FB, Stampfer MJ, Manson JE, Willett WC. Major dietary protein sources and risk of coronary heart disease in women. Circulation. 2010;122(9):876-83.

30. Tian S, Xu Q Jiang R, Han T, Sun C, Na L. Dietary Protein Consumption and the Risk of Type 2 Diabetes: A Systematic Review and Meta-Analysis of Cohort Studies. Nutrients. 2017;9(9). pii:E982. 
31. Wu J, Zeng R, Huang J, Li X, Zhang J, Ho JC-M, et al. Dietary Protein Sources and Incidence of Breast Cancer: A DoseResponse Meta-Analysis of Prospective Studies. Nutrients. 2016;8(11). pii:E730.

32. Steell L, Sillars A, Welsh P, Iliodromiti S, Wong SC, Pell JP, et al. Associations of dietary protein intake with bone mineral density: An observational study in 70,215 UK Biobank participants. Bone. 2019;120:38-43.

33. Mangano KM, Sahni S, Kerstetter JE. Dietary protein is beneficial to bone health under conditions of adequate calcium intake: an update on clinical research. Curr Opin Clin Nutr Metab Care. 2014 J;17(1):69-74.

34. de Boer J, Aiking H. Prospects for pro-environmental protein consumption in Europe: Cultural, culinary, economic and psychological factors. Appetite. 2018;121:29-40.

35. Willett W, Rockström J, Loken B, Springmann M, Lang T, Vermeulen S, et al. Food in the Anthropocene: the EATLancet Commission on healthy diets from sustainable food systems. Lancet. 2019; 393(10170): 447-492.
36. Clayton S, Myers G. Conservation Psychology: Understanding and Promoting Human Care for Nature. Segunda edición. Wiley-Blackwell; 2015.

37. Flora P. State formation, nation building and mass politics in Europe. The theory of Stein Rokkan on his collected works. Primera edición. Oxford: Claredon Press, editor; 1999.

38. Alexander P, Brown C, Arneth A, Dias C, Finnigan J, Moran $\mathrm{D}$, et al. Could consumption of insects, cultured meat or imitation meat reduce global agricultural land use? Glob Food Sec. 2017;15:22-32.

39. Jones OG. Recent advances in the functionality of non-animal-sourced proteins contributing to their use in meat analogs. Curr Opin Food Sci. 2016;7:7-13.

40. Auestad N, Fulgoni VL. What Current Literature Tells Us about Sustainable Diets: Emerging Research Linking Dietary Patterns, Environmental Sustainability, and Economics. Adv Nutr. 2015;6(1):19-36.

41. Nelson ME, Hamm MW,Hu FB, Abrams SA, Griffin TS. Alignment of Healthy Dietary Patterns and Environmental Sustainability: A Systematic Review. Adv Nutr. 2016;7(6):1005-25. 